\title{
Влияние электрического поля на энергию активации локальных уровней в полупроводниках со слоистой (GaSe) и кубической структурой $\left(\mathrm{Ga}_{2} \mathrm{Se}_{3}\right)$
}

\author{
(C) А.М. Пашаев ${ }^{1}$, Б.Г. Тагиев ${ }^{1,2, \text { ฯ О.Б. Тагиев }}{ }^{2,3}$, В.Т. Межидова ${ }^{1}$, И.3. Садыхов ${ }^{1}$ \\ ${ }^{1}$ Национальная академия авиации, \\ Az-1045 Баку, Азербайджан \\ ${ }^{2}$ Институт ффизики Национальой академии наук Азербайджана, \\ Az-1143 Баку, Азербайджан \\ ${ }^{3}$ Филиал Московского государственного университета им. М.В. Ломоносова, \\ Az-1143 Баку, Азербайджан \\ ^E-mail: bahadur34@mail.ru
}

(Получена 25 апреля 2018 г. Принята к печати 6 июня 2018 г.)

\begin{abstract}
Представлены результаты измерений электропроводности слоистых кристаллов (GaSe, GaTe и их твердых растворов) и кубических кристаллов типа $\mathrm{Ga}_{2} \mathrm{Se}_{3}$ в сильных электрических полях до $5 \cdot 10^{5} \mathrm{~B} / \mathrm{cm}$ в интервале температур (77-300)К. Полученные результаты были сравнены с феноменологической теорией концентрационной неустойчивости в полупроводниках. В этой теории рассматривается роль эффекта Френкеля, связанного с термоэлектронной ионизацией ловушек, приводящих к процессу неустойчивости в полупроводниках с $S$-образной вольт-амперной характеристикой. На основании результатов измерений электропроводности слоистых и кубических кристаллов с эффектом Френкеля и теорией неустойчивости тока в полупроводниках оценена концентрация свободных носителей тока в указанных типах халькогенидных полупроводников: $n=\left(3 \cdot 10^{13}-4 \cdot 10^{15}\right) \mathrm{cm}^{-3}$.
\end{abstract}

DOI: 10.21883/FTP.2019.02.47102.8676

\section{1. Введение}

Фундаментальными параметрами полупроводников и диэлектриков являются ширина запрещенной зоны $E_{g}$, энергия активации доноров $E_{d}$ (или акцепторов $E_{a}$ ) и др., которые определяются электрофизическими и оптическими методами [1-5]. Известно, что один из способов определения ширины запрещенной зоны и энергии активации локальных уровней в полупроводниках основан на температурной зависимости электропроводности в собственной и примесных областях проводимости. Теоретические и экспериментальные исследования показывают, что как ширина запрещенной зоны полупроводников, так и энергия активации локальных уровней зависят от внешних факторов. Такими факторами являются сильное электрическое поле (эффекты Френкеля [6], Франца-Келдыша [7], ударная ионизация [8] и др.), температура, которые влияют на ширину запрещенной зоны и энергию активации ловушек. Причины изменения запрещенной зоны и энергии активации локальных уровней хорошо объясняются в литературе (см., например, $[9,10])$. Зависимость электропроводности $(\sigma=e n \mu)$ от температуры, сильного электрического поля, интенсивности света и других внешних факторов связана с изменением концентрации носителей тока $n$ и их подвижности $\mu$. Теория и эксперименты показывают, что во многих случаях концентрация носителей тока $n$ сильнее зависит от величины внешних факторов (например, от температуры), чем от их подвижности. Исходя из этих соображений можно заключить, что температурная зависимость электропроводности полупроводников в основном определяется температурной зависимостью концентрации носителей тока. Это величина экспоненциально зависит от температуры

$$
n=n_{0} e^{\frac{-E_{t}}{k T}},
$$

где $n_{0}-$ концентрация носителей тока при, $\frac{10^{3}}{T} \rightarrow 0$, $E_{t}$ - энергия активации локальных уровней.

Увеличение концентрации свободных носителей тока в зависимости от электрического поля, температуры и концентрации примесных уровней приводит к сдвигу и исчезновению локальных уровней [6-12]:

$$
\begin{gathered}
E_{t}=E_{0}-e \sqrt{\frac{e E}{\pi \varepsilon \varepsilon_{0}}}, \\
E_{t}=E_{0}-\frac{n}{\bar{n}} k T .
\end{gathered}
$$

Цель настоящей работы - исследовать электропроводность образцов слоистых кристаллов типа GaSe и кубических кристаллов типа $\mathrm{Ga}_{2} \mathrm{Se}_{3}$ в сильных электрических полях до $5 \cdot 10^{5} \mathrm{~B} / \mathrm{cm}$ в интервале температур (77-300) К и оценить концентрацию носителей тока на основании сопоставления формулы (2) и (3) с экспериментальными данными.

\section{2. Результаты измерений и их обсуждения}

Монокристаллы халькогенидов галлия были выращены по видоизмененному методу Бриджмена медленным охлаждением слитка при постоянном градиенте 
температуры [13]. Исходными веществами служили селен марки В-5 (99.99999\%) галлий и теллур марки В-3 (99.99999\%). Синтез соединений $\mathrm{GaSe}, \mathrm{GaTe}$, $\mathrm{GaSe}_{x} \mathrm{Te}_{1-x}$ (где $x=1.00,0.95,0.90,0.80,0.7,0.30$, $0.20,0.10,0)$ и $\mathrm{Ga}_{2} \mathrm{Se}_{3}, \mathrm{GaTe}_{3}$ и выращивание их монокристаллов проведены в откачанных до $10^{-4}$ мм рт. ст. остроконечных кварцевых ампулах.

Методы синтеза, выращивания монокристаллов соединений со слоистой и кубической структурами, принадлежащих к обширному классу группы $\mathrm{A}^{\mathrm{III}} \mathrm{B}^{\mathrm{VI}}(\mathrm{GaSe}$, İnSe, GaS, İnS, и др) и $\mathrm{A}_{2}^{\mathrm{III}} \mathrm{B}_{3}^{\mathrm{VI}}\left(\mathrm{Ga}_{2} \mathrm{~S}_{3}, \mathrm{Ga}_{2} \mathrm{Se}_{3}, \mathrm{Ga}_{2} \mathrm{Te}_{3}\right.$ и др.), а также изготовление образцов для электрических измерений в области сильных электрических полей описаны в [14-16].

Полученные кристаллы подвергались рентгенографическому исследованию с целью проверки их монокристалличности. Лауэграммы показали монокристалличность вышеуказанных кристаллов; были вычислены параметры их решеток. Анализ дифрактограмм, полученных от поликристаллических образцов $\mathrm{GaSe}_{x} \mathrm{Te}_{1-x}$, показывает, что твердые растворы со стороны GaTe сохраняют симметрию моноклинной, а со стороны $\mathrm{GaSe}$ - гексагональной решетки. Полученные монокристаллы были оптически однородными, высокоомными $\left(\sim 10^{8} \mathrm{OM} \cdot \mathrm{M}\right)$, фоточувствительными и имели форму цилиндра высотой 80 мм и диаметром до 20 мм.

Электропроводность монокристаллов $\mathrm{GaSe}, \mathrm{GaTe}$ и их твердых растворов, а также $\mathrm{Ga}_{2} \mathrm{Se}_{3}$ и $\mathrm{Ga}_{2} \mathrm{Te}_{3}$ была измерена в сильных импульсных электрических полях [14].

В выражениях (2) и (3) $E_{0}$ - энергия активации при отсутствии внешних факторов, $E-$ величина электрического поля, при котором наблюдается зависимость $E_{t}$ от $T$ и $E$. В (3) $\bar{n}=m \varepsilon(k T)^{2} / 2 \pi \hbar^{2} q^{2}-$ характеристическая концентрация, уменьшающая $E_{0}$ на $k T$. Формула (2) впервые получена Френкелем, и авторами [12] использована для объяснений неустойчивости полупроводников с $S$-образной вольт-амперной характеристикой (BAX).

В последние годы эффект Френкеля используется для объяснения влияния сильного электрического поля на токопрохождение в полупроводниках и структурах на их основе [17-20].

На рис. 1 и 2 при разных температурах представлены результаты измерений электропроводности образцов слоистых кристаллов GaSe и GaTe (рис. 1,a,b) и кубических кристаллов типа $\mathrm{Ga}_{2} \mathrm{Se}_{3}$ (рис. 2). Видно, что с уменьшением температуры величина поля $E$, при котором закон Ома нарушается, увеличивается в обоих типах кристаллов. Измерения эффекта Холла [21-23] показывают, что в интервале температур $167-287 \mathrm{~K}$, в котором снята зависимость $\sigma(E)$, подвижность носителей тока с уменьшением температуры возрастает. Если нарушение закона Ома обусловлено разогревом носителей тока, то критическое поле, при которым закон Ома нарушается, должно было уменьшаться с понижением температуры. Из рис. $1, a, b$ и 2 видно, что в указанных слоистых и кубических кристаллах
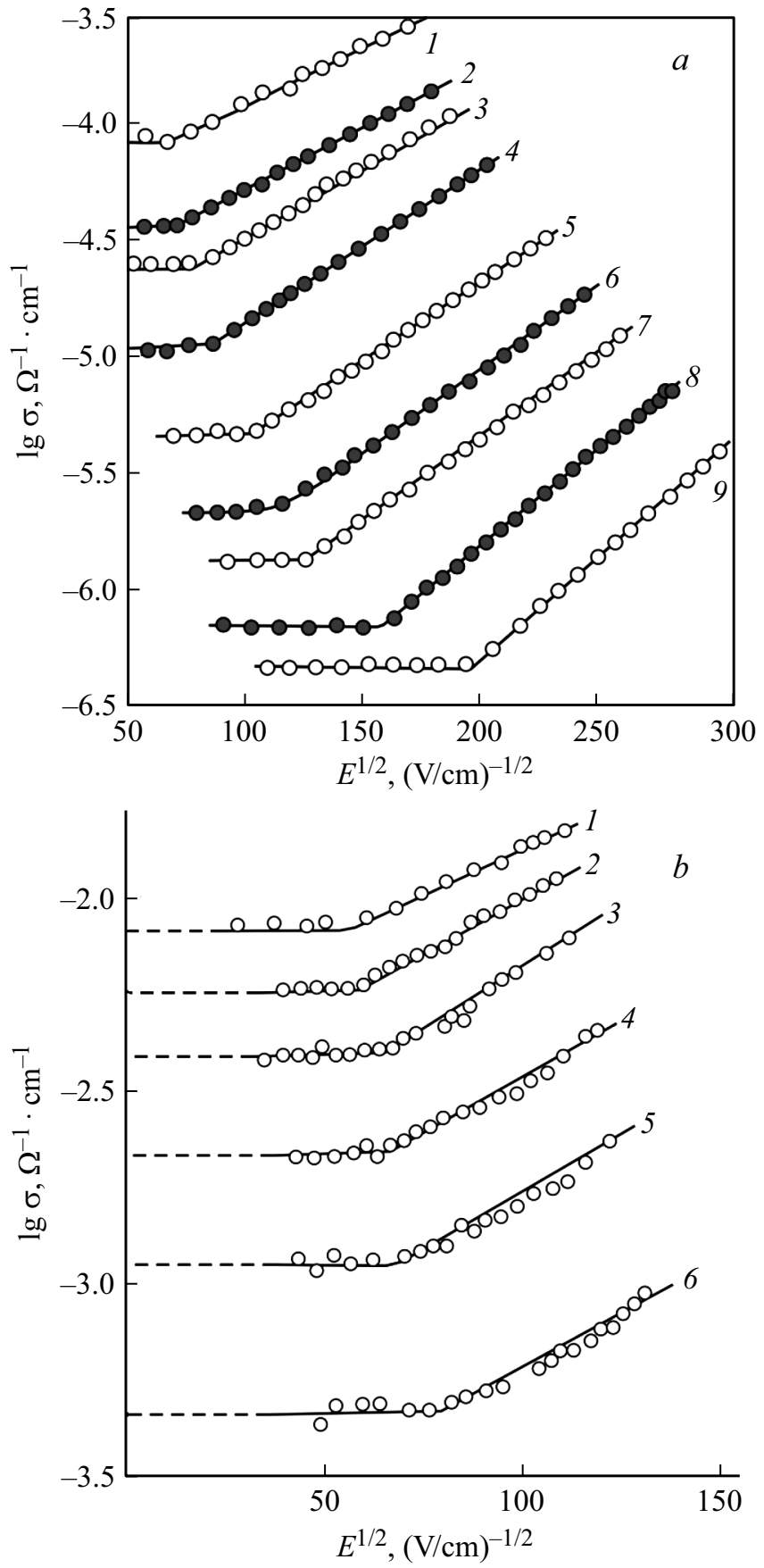

Рис. 1. $a$ - зависимость электропроводности $\left(\sigma, \mathrm{OM}^{-1} \cdot \mathrm{cm}^{-1}\right)$ образца $\mathrm{GaSe}$ от напряженности электрического поля $(E, \mathrm{~B} / \mathrm{cm})$ при различных температурах $(T, \mathrm{~K}): 1-285,2-270$, $3-258,4-246,5-230,6-217,7-204,8-186,9-$ 167. $b$ - зависимость электропроводности $\left(\sigma, \mathrm{OM}^{-1} \cdot \mathrm{cm}^{-1}\right)$ образца $\mathrm{GaTe}$ от напряженности электрического поля $(E, \mathrm{~B} / \mathrm{cm})$ при различных температурах $(T, \mathrm{~K}): 1-232,2-217$, $3-202,4-185,5-170,6-150$.

этот факт не подтверждается. Поэтому, как отмечается в [24], рост критического поля, при котором закон Ома нарушается с понижением температуры, связан с уменьшением вероятности термического возбуждения. 


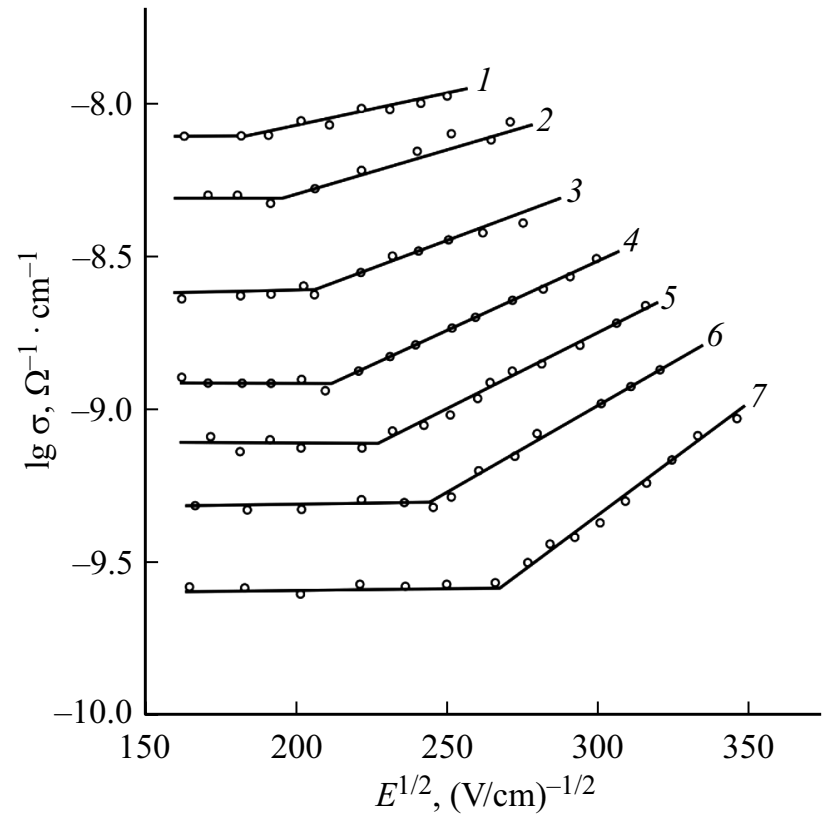

Рис. 2. Зависимость электропроводности монокристалла $\mathrm{Ga}_{2} \mathrm{Se}_{3}$ от напряженности электрического поля $E$ при различных температурах $(T, \mathrm{~K}): 1-250,2-224,3-200,4-190$, $5-150,6-110,7-83$.

В слоистых кристаллах GaSe, GaTe и их твердых растворах наличие ковалентной связи внутри и ван-дерваальсовой связи между слоями приводит к анизотропии их физических свойств, что вызвало интерес к исследованию электропроводности $\sigma$ в двух направлениях $\left(\sigma_{\|}\right.$- параллельно и $\sigma_{\perp}-$ перпендикулярно слоям).

Результаты измерений анизотропии электропроводности $\sigma_{\|} / \sigma_{\perp}$ для монокристаллов GaSe представлены на рис. 3. Видно, что $\sigma$ в направлении, перпендикулярном слоям, в десятки раз меньше, чем в направлении, параллельном слоям. Аналогичные зависимости имеют место для всех твердых растворов исследуемых составов $\mathrm{GaSe}_{x} \mathrm{Te}_{1-x}$. При этом установлено, что энергия активации носителей тока в направлении, перпендикулярном слоям, превышает энергию активации в направлении, параллельном слоям. Поэтому можно предположить, что энергия активации $E_{t}$ ловушек носителей тока в $\mathrm{GaSe}, \mathrm{GaTe}$ и в других слоистых полупроводниках, определенная из температурной зависимости электропроводности, в направлении, перпендикулярном слоям, равна сумме энергий активации носителей тока внутри слоя $\left(E_{\|}\right)$и высоты барьера $\left(E_{\delta}\right)$ между слоями:

$$
E_{\perp}=E_{\|}+E_{\delta}
$$

Таким образом, можем написать, что

$$
\begin{gathered}
\sigma_{\perp} \sim \exp \frac{E_{\|}+E_{\delta}}{k T}, \\
\sigma_{\|} \sim \exp \left(\frac{E_{\|}}{k T}\right) .
\end{gathered}
$$

Из (5) и (6) имеем

$$
\frac{\sigma_{\|}}{\sigma_{\perp}} \sim \exp \left(\frac{E_{\delta}}{k T}\right) .
$$

В соответствии с уравнением (7) с понижением температуры отношение $\sigma_{\|} / \sigma_{\perp}$ увеличивается и при определенной температуре, величина которой зависит от состава, достигает насыщения. По данным, представленным на рис. 3, оценена высота барьера между слоями, равная $E_{\delta}=(0.03+0.10)$ эВ для твердых растворов $\mathrm{GaSe}_{x} \mathrm{Te}_{1-x}$. Полученные значения для $E_{\delta}$ хорошо согласуются с результатами измерений холловской подвижности $[21-23,25]$.

Как видно из рис. $1, a, b$ и рис. 2 , линейная зависимость между $\lg \sigma$ и $\sqrt{E}$ подтверждает, что в кристаллах $\mathrm{GaSe}_{x} \mathrm{Te}_{1-x}$ и $\mathrm{Ga}_{2} \mathrm{Se}_{3}$, в соответствии с формулой (3), рост электропроводности с увеличением $E$ обусловлен термоэлектронной ионизацией Френкеля. Согласно теории Френкеля, наклон $\beta$ зависимости $\lg \sigma$ от $\sqrt{E}$ с понижением температуры увеличивается:

$$
\beta=\frac{\sqrt{e^{3}}}{k T \sqrt{\pi \varepsilon_{0} \varepsilon_{\infty}}},
$$

где $e-$ заряд электрона, $\varepsilon_{0}=8.85 \cdot 10^{-12} \Phi / \mathrm{M}-$ электрическая постоянная, $\varepsilon_{\infty}=n^{2}(n-$ коэффициент преломления материала) - высокочастотная проницаемость полупроводника и диэлектрика.

Выражение (8) позволяет по экспериментальным данным определить высокочастотную диэлектрическую проницаемость $\varepsilon_{\infty}$.

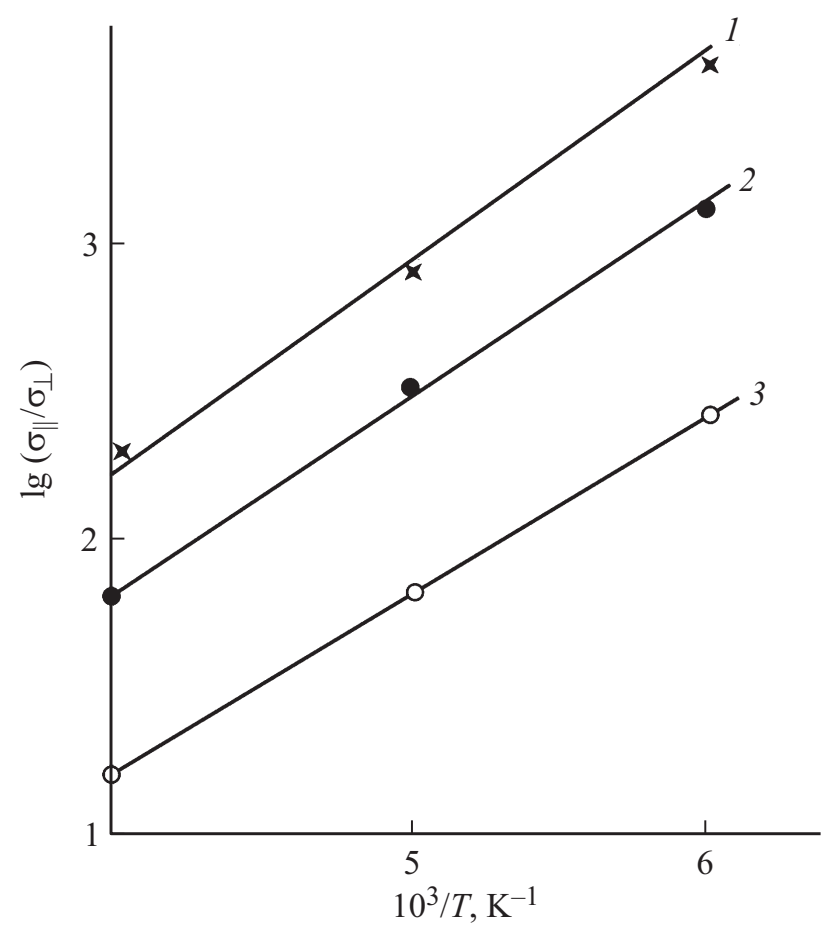

Рис. 3. Температурная зависимость $\lg \frac{\sigma_{\|}}{\sigma_{\perp}}$ монокристалла GaSe при различных полях $(E$, кВ/см): $1-17,2-16,3-13$. 


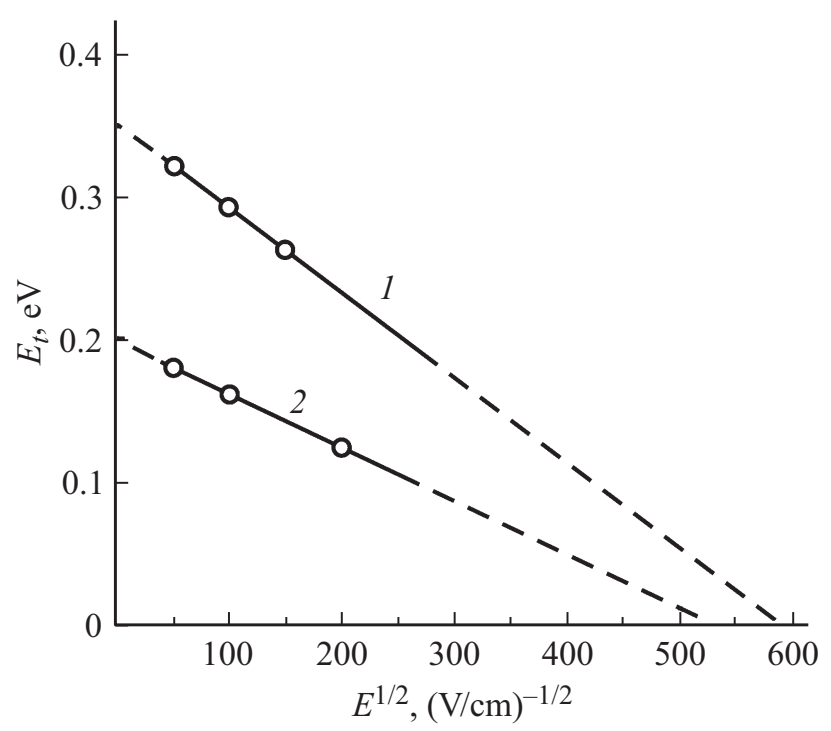

Рис. 4. Зависимость энергии активации $E_{t}$ ловушек в монокристалле $\mathrm{Ga}_{2} \mathrm{Se}_{3}(1)$ и $\mathrm{GaSe}(2)$.

Подставляя экспериментальные данные для $\beta$ в (8) были определены значения $\varepsilon_{\infty}$ в слоистых кристаллах $\operatorname{GaSe}\left(\varepsilon_{\infty}^{\|}=10, \varepsilon_{\infty}^{\perp}=8\right)$, GaTe $\left(\varepsilon_{\infty}^{\|}=7, \varepsilon_{\infty}^{\perp}=6\right)$ и в кубических кристаллах $\mathrm{Ga}_{2} \mathrm{Se}_{3}\left(\varepsilon_{\infty}=10.8\right), \mathrm{Ga}_{2} \mathrm{Te}_{3}$ $\left(\varepsilon_{\infty}=11.5\right)$.

Следует подчеркнуть, что эффект сильного электрического поля в полупроводниках, в которых наблюдается закон Френкеля, имеет преимущество перед другими методами при определении $\varepsilon$, в частности ее электронной части $\varepsilon_{\infty}$. Измерение $\varepsilon$ полупроводников с удельным сопротивлением $\left(10^{2}-10^{6}\right)$ Ом $\cdot$ см емкостным методом сопровождается диэлектрической потерей.

Что касается оптического метода, то здесь трудность связана с определением коэффициента преломления в направлении, перпендикулярном слоям. Выполнение закона Френкеля в полупроводниках позволяет легко определить электронную часть диэлектрической проницаемости в слоистых материалах как вдоль, так и поперек слоев. Отметим, что знание величины $\varepsilon$ играет важную роль при интерпретации оптических свойств полупроводников и при определении емкости в полупроводниковых преобразователях, в частности $p-n$-переходах и переключающих приборах.

На основе результатов измерений зависимости $\sigma$ от $\sqrt{E}$, представленных на рис. $1, a, b$ и 2 , построена зависимость кубических кристаллов $\sigma$ от $10^{3} / T$ при различных электрических полях. Анализ полученных данных показывает, что как в области выполнения закона Ома, так и в сильных полях имеет место закономерность (3). При этом обнаружено уменьшение наклона прямых $\lg \sigma$ от $10^{3} / T$, т. е энергии активации $E_{t}$ в зависимости от $E$. Уменьшение $E_{t}$ при различных полях согласуется с выражением (4). Эта закономерность демонстрируется на рис. 4, где представлены зависимости $E_{t}$ от $E$ для монокристаллов $\mathrm{GaSe}$ и $\mathrm{Ga}_{2} \mathrm{Se}_{3}$ (кривые 1 и 2 соот- ветственно). Экстраполяция кривых 1 и 2 для GaSe и $\mathrm{Ga}_{2} \mathrm{Se}_{3}$ соответственно в сторону увелечения электрического поля позволила определить поле пробоя для этих кристаллов, которые оказались равными $5 \cdot 10^{5}$ и $7 \cdot 10^{5} \mathrm{~B} / \mathrm{cм}$. При этих полях

$$
E_{0}-e \cdot \sqrt{\frac{e E}{\pi \varepsilon \varepsilon_{0}}}=0, \quad E_{0}=e \sqrt{\frac{e E}{\pi \varepsilon \varepsilon_{0}}} .
$$

Отсюда следует вывод, что, когда в полупроводниках и диэлектриках локальные уровни полностью ионизируются, т. е исчезают, имеет место равенство

$$
e \sqrt{\frac{e E}{\pi \varepsilon \varepsilon_{0}}}=\frac{n}{\bar{n}} k T .
$$

Оценка $\quad \bar{n}=m \varepsilon(k T)^{2} / 2 \pi \hbar^{2} q^{2}$ по известным физическим величинам $\left(m=9.1 \cdot 10^{-31}\right.$ кг - эффективная масса электрона, $\varepsilon=\varepsilon_{0} \varepsilon_{\infty}=10 \cdot 8.85 \cdot 10^{-12} \Phi / \mathrm{M}$, $k=1.38 \cdot 10^{-23}$ Дж/K, $T=300 \mathrm{~K}, \hbar=1.05 \cdot 10^{-34}$ Дж $\cdot \mathrm{c}$, $q=1,6 \cdot 10^{-19}$ Кл), величина которой равна $\bar{n}=$ $=7.3 \cdot 10^{17} \mathrm{~cm}^{-3}$, позволила из (8) определить концентрацию свободных носителей тока в слоистых кристаллах $\mathrm{GaSe}_{x} \mathrm{Te}_{1-x}\left(4 \cdot 10^{14}-5 \cdot 10^{13}\right) \mathrm{cm}^{-3}$ и в кубических кристаллах $\mathrm{Ga}_{2} \mathrm{Se}_{3}\left(5 \cdot 10^{8}-10^{10} \mathrm{~cm}^{-3}\right)$.

\section{3. Заключение}

Анализ результатов измерений электропроводности слоистых $\mathrm{GaSe}_{x} \mathrm{Te}_{1-x}$ и кубических $\mathrm{Ga}_{2} \mathrm{Se}_{3}$ кристаллов показывает, что наклон температурной зависимости $\lg \sigma=f\left(\frac{10^{3}}{T}\right)$ с увеличением $E$ уменьшается в соответствии с формулой (2) и при этом $E_{t}$ линейно уменьшается с ростом $\sqrt{E}$. Экстраполяция этой зависимости в строну увеличения электрического поля позволила определить величину электрического поля пробоя в слоистых (типа GaSe) и кубических (типа $\mathrm{Ga}_{2} \mathrm{Se}_{3}$ ) кристаллах, которая находится в интервале $\left(5 \cdot 10^{5}-7 \cdot 10^{5}\right) \mathrm{B} / \mathrm{cm}$. Совместно решая уравнение $(3)$ и (4) с учетом экспериментальных данных об электропроводности в зависимости от электрического поля, определена концентрация избыточных носителей тока, равная $\left(4 \cdot 10^{14}-5 \cdot 10^{13}\right) \mathrm{cm}^{-3}$ и $\left(5 \cdot 10^{8}-10^{10}\right) \mathrm{cm}^{-3}$ для кристаллов типа GaSe и кубических кристаллов типа $\mathrm{Ga}_{2} \mathrm{Se}_{3}$ соответственно.

\section{Список литературы}

[1] Ф. Мейзда. Электронные измерительные приборы и методы измерений (М., Мир, 1990).

[2] А.А. Рогачев, И.Н. Саблина. ФТТ, 8 (1), 187 (1966).

[3] К.В. Шалимова. Практикум по полупроводникам и полупроводниковым приборам (М., Высш. шк., 1968).

[4] И.Ю. Уханов. Оптические свойства полупроводников (М., Наука, 1977).

[5] В.И. Фистуль. Введение в физику полупроводников (М., Высш. шк., 1984). 
[6] Я.И. Френкель. ЖЭТФ, 8, 1893 (1938).

[7] В.Л. Бонч-Бруевич, С.Г. Калашников. Физика полупроводников (М., Мир, 1977).

[8] Ф. Блатт. Теория подвижности электронов в твердых meлаx (М., Физматгиз, 1963).

[9] Я.И. Френкель. ЖЭТФ, 23, 619 (1952).

[10] А.М. Саржевский. Оптика. Полный курс (Едиториал УРCC, 2004).

[11] И.3. Фишер, Ч.К. Смолик. ФТТ. Сб. статей, ч. 2 (М., 1959).

[12] В.Б. Сандомирский, А.А. Суханов, А.Г. Ждан. ЖЭТФ, 58 (5), 1683 (1970).

[13] 3.С. Медведева. Халькогениды элементов ІІІ (Б) подгруппы Периодической системы (М., Наука, 1968).

[14] E.S. Guseinova, V.A. Gadzhiev, B.G. Tagiyev. Phys. Status Solidi B, 36, 75 (1969).

[15] А.М. Пашаев, Б.Г. Тагиев, О.Б. Тагиев. ФТТ, 55 (5), 861 (2013).

[16] А.Ф. Иоффе. Физика полупроводников (М.; Л., Изд-во AH CCCP, 1957).

[17] S.D. Ganichev, I.N. Yassievich, A.A. Istratov, Eicke R. Weber. Phys. Rev. B, 61 (15), 361 (2000).

[18] К.А. Насыров, В.А. Гриценко, Ю.Н. Новиков, Д.В. Гриценко, Д.-В. Ли, Ч.В. Ким. Изв. РГПУ им. А.И. Герцена., №5 (13),447 (2005).

[19] Y.Q. Wu, H.C. Lin, P.D. Ye. Appl. Phys. Lett., 90 (7), 072105 (2007).

[20] В.А. Гриценко. УФН, 182 (5), 531 (2012).

[21] C. Manfredotti, R. Murri, A. Rizzo. Phys. Rev. B, 10 (8), 3387 (1974).

[22] C. Manfredotti, A.M. Mancini, R. Murri, A. Rizzo, L. Vasenelli. Nuovo Cimento B, 39 (1), 257 (1977).

[23] А.П. Одринский. ФТП, 44, 883 (2010).

[24] G.A. Dussel, K.W. Boer. Phys. Status Solidi B, 39, 375 (1970).

[25] Б.Н. Брудный, А.В. Кособуцкий, С.Ю. Саркисов. ФТП, 44, 1194 (2010).

Редактор Г.А. Оганесян

\section{Influence of electric field on the activation energy of local levels in semiconductors with layered (GaSe) and cubic structure $\left(\mathrm{Ga}_{2} \mathrm{Se}_{3}\right)$}

A.M. Pashayev ${ }^{1}$, B.H. Tagiyev ${ }^{1,2}$, O.B. Tagiyev ${ }^{2,3}$, V.T. Majidova ${ }^{1}$, I.Z. Sadikhov ${ }^{1}$

${ }^{1}$ National Aviation Academy, Az-1045 Baku, Azerbaijan

${ }^{2}$ Azerbaijan National Academy of Sciences, Institute of Physics,

Az-1143 Baku,Azerbaijan

${ }^{3}$ Lomonosov Moscow State University Campus

in Baku,

Az-1143 Baku, Azerbaijan

Abstract The results of electro-conductivity in high electric fields (up to $5 \cdot 10^{5} \mathrm{~V} / \mathrm{cm}$ ) in temperature range of $(77-300) \mathrm{K}$ are presented for layered (GaSe, GaTe and their solid solutions) and cubic $\mathrm{Ga}_{2} \mathrm{Se}_{3}$ crystals. Obtained results were compared with the phenomenological theory of concentration instability in semiconductors. Role of Frenkel's effect, connected with thermo-electronic ionization of traps leading to instability processes in semiconductors with $S$-type CV characteristics are considered. Based on the results of electro-conductivity measurements of layered and cubic crystals with Frenkel's effect and theory of current instability in semiconductors the free currier concentrations are estimated for mentioned semiconductors: $n=\left(3 \cdot 10^{13}-4 \cdot 10^{15}\right) \mathrm{cm}^{-3}$. 